\title{
Use of posaconazole in the treatment of infective rhinocerebral mucormycosis
}

\author{
K Kulendra, M Habibi*, C Butler, P Clarke $\dagger$, D Howard $\dagger$
}

\begin{abstract}
Introduction: A patients with undiagnosed type II diabetes mellitus presented with infective rhinocerebral mucormycosis.

Investigation results: Initial magnetic resonance imaging scans demonstrated an aggressive disease process involving the left orbit and paranasal sinuses. A repeated scan following treatment excluded intracranial spread or recurrence. Pus from the paranasal sinuses grew Rhizopus arrhizus on microbiological culture.

Management: Initial treatment comprised intravenous liposomal amphotericin B, intravenous co-amoxiclav and surgical debridement. The patient's diabetes was managed medically. The development of drug-induced transaminitis required a change of medication. The dose of liposomal amphotericin B was reduced, and then titrated back up as the liver function test results improved. Posaconazole was also introduced and the patient was eventually discharged on this alone, as the maximum recommended cumulative dose of liposomal amphotericin B had been reached.

Conclusions: Posaconazole may be used effectively in conjunction with surgical debridement in the treatment of patients with infective rhinocerebral mucormycosis who develop hepatotoxic side effects to liposomal amphotericin B. Posaconazole may also allow a reduction in the dose of liposomal amphotericin B, resulting in better tolerance.
\end{abstract}

Key words: Mucorymycosis; Paranasal Sinuses; Brain; Amphotericin B; Posaconazole; Otorhinolaryngologic Surgical Procedures

\section{Introduction}

Mucormycosis is an invasive fungal disease caused primarily by fungi from the subphylum mucormycotina and the order mucorales. ${ }^{1-4}$ Four of the fourteen families are pathogenic, with mucoraceae being most significant. Patients with mucor commonly have organisms related to this genus, including rhizopus, rhizomucor and absidia. ${ }^{3}$ These organisms are found in soil and are important in the decay of organic material. The presence of such opportunistic pathogens suggests a significant underlying predisposing factor. The most common form of rhinocerebral mucormycosis is characterised by progressive fungal invasion of the paranasal sinuses, orbit, hard palate and brain.

The cornerstone of mucormycosis therapy is combined treatment with surgical debridement plus prolonged, high-dose $(\geq 5 \mathrm{mg} / \mathrm{kg})$ liposomal amphotericin $\mathrm{B}$, as treatment failure occurs when antifungals are used in isolation. ${ }^{5}$ However, intolerance to the high doses of liposomal amphotericin B required can result in inadequate therapy. Posaconazole, a newer, oral, triazole antifungal, is well tolerated and its use in mucormycosis salvage therapy has previously been described. $^{6,7}$ Animal data suggests that combination therapy with liposomal amphotericin B and posaconazole may allow reduction in liposomal amphotericin B dosage, potentially resulting in fewer adverse effects. 8

Here, we describe the treatment of rhinocerebral mucormycosis with posaconazole combined with reduced-dose liposomal amphotericin $\mathrm{B}$, in addition to surgical debridement, followed by monotherapy with posaconazole, in a patient with drug-induced transaminitis following highdose liposomal amphotericin B treatment.

\section{Case report}

A 59-year-old man was referred by his local ENT department with his initial magnetic resonance imaging scans (Figure 1) for treatment of confirmed infective rhinocerebral mucormycosis. He had initially presented six weeks previously to primary care with a dull headache and leftsided facial hypoaesthesia. He had then consulted his optician complaining of further symptoms, including diplopia, proptosis and deteriorating vision in his left eye. This had progressed to complete visual loss and deteriorating hearing on the left side, prior to his admission to Charing Cross Hospital.

On examination, the patient's left eye was proptosed, with complete ptosis. Cranial nerves II, III, IV and VI were not functioning on the left side, and the left pupil was fixed and dilated. A dull, red tympanic membrane was noted in the left ear. The oral cavity was unremarkable. Flexible nasal endoscopy revealed green crusting within the left nasal cavity .

An urgent computed tomography scan of the sinuses was performed (Figure 2), demonstrating an aggressive disease process involving the medial wall of the left orbit, left 


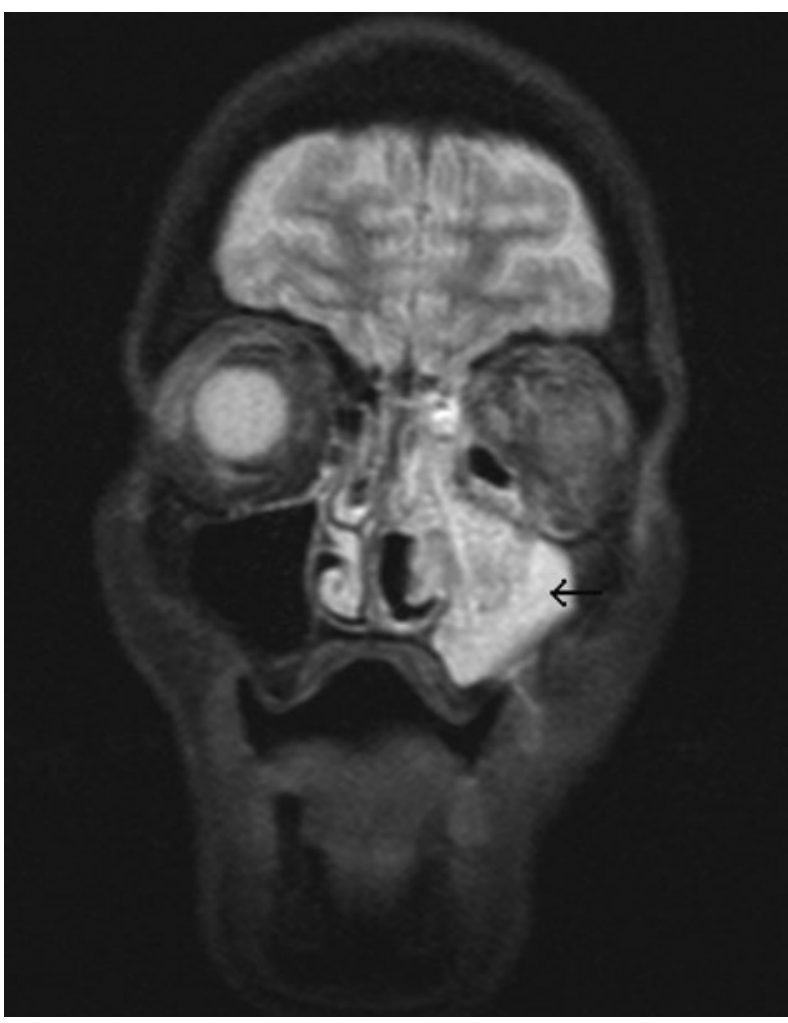

FIG. 1

Coronal magnetic resonance imaging scan of the head, showing invasive fungal sinusitis (arrow) affecting the left paranasal sinuses. Proptosis of the left eye is also present as the globe has been displaced anteriorly.

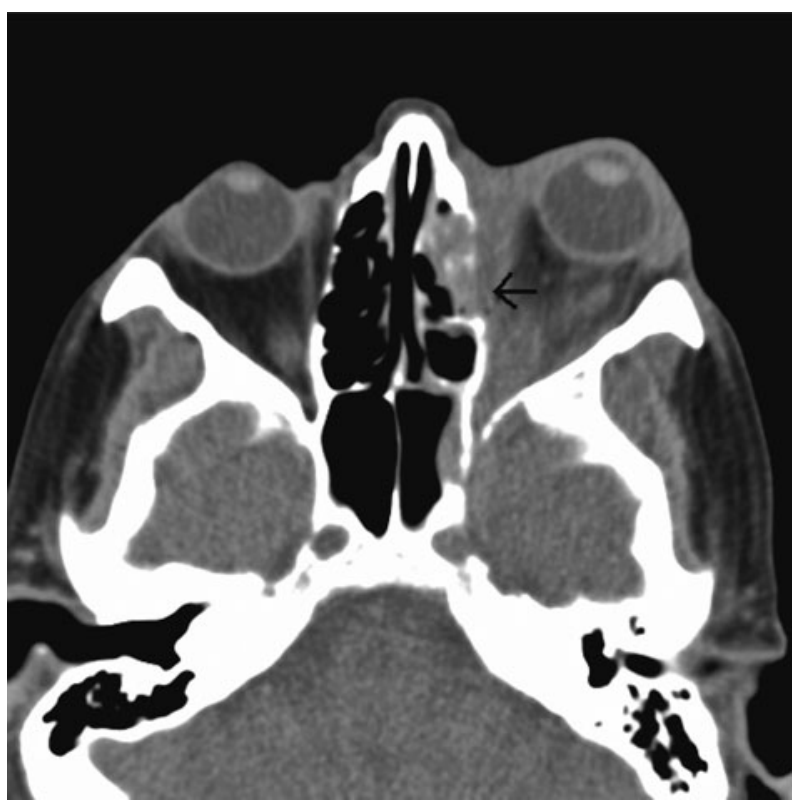

FIG. 2

Axial computed tomography scan of the head, showing fungal sinusitis invading the left orbit via the paranasal sinuses (arrow) and causing left-sided proptosis.

ethmoid, and maxillary and sphenoid sinuses, with extension into the pterygopalatine and infratemporal fossae.

Following consultation with local infectious disease specialists, treatment was commenced with intravenous liposomal amphotericin B (AmBisome ${ }^{\circledR}$; Astellas Pharma, Deerfield, USA). An initial test dose of $5 \mathrm{mg} / \mathrm{kg}$ was given. Empirical antibiotic therapy with intravenous coamoxiclav was also administered to cover bacterial sinusitis.

The patient underwent radical surgical debridement that evening. Left lateral rhinotomy, maxillectomy and orbital exenteration were performed. Pus was discovered in the ethmoid and sphenoid sinuses, and microbiological culture later confirmed the growth of Rhizopus arrhizus. Ethmoidectomies, maxillectomy and sphenoidectomy were conducted, and the surgeons proceeded to orbital exenteration as necrotic tissue was discovered in the orbital apex, inferior orbital fissure and skull base.

The patient was found to have an elevated fasting glucose level of $21.3 \mathrm{mmol} / 1$ with associated glycosuria, confirming the diagnosis of diabetes mellitus. He was reviewed by local diabetologists and treated in the initial period with oral hypoglycaemics.

On the third day of liposomal amphotericin B therapy, the patient was found to have elevated alanine transaminase levels (2002 IU/1). The co-amoxiclav was discontinued and the dose of liposomal amphotericin $\mathrm{B}$ reduced to $1 \mathrm{mg} / \mathrm{kg}$. Following discussion with local specialist infectious disease physicians and microbiologists, oral posaconazole was commenced at a dose of $400 \mathrm{mg}$ twice daily, once liver function test results were within normal limits. Following 10 days of reduced-dosage liposomal amphotericin B therapy, the liposomal amphotericin $\mathrm{B}$ dosage was then increased up to a maximum of $10 \mathrm{mg} / \mathrm{kg}$.

When a further left-sided deterioration in hearing was reported, examination suggested left-sided sensorineural hearing loss, with a positive Rinne's test bilaterally and Weber's test localising to the right side. Pure tone audiometry confirmed a left sensorineural hearing loss, with thresholds of $75-105 \mathrm{~dB}$. Following intratympanic steroid injection, repeated audiometry indicated minimal improvement (threshold of 70-85 dB). It was not clear whether the patient's hearing loss was secondary to disease progression or medication.

The patient was taken back to the operating theatre for examination under anaesthesia and decrusting of the cavity, in order to improve aeration. Necrotic bone was noted in the cribriform plate and frontal recess. An urgent magnetic resonance imaging (MRI) head scan was therefore arranged, which demonstrated no intracranial spread. This was discussed in a multidisciplinary meeting; following comparison with previous scans, it was concluded that there had been no disease progression.

After a six-week stay in hospital, the patient was approaching the maximum recommended cumulative dose of liposomal amphotericin B (i.e. $16.8 \mathrm{~g}$ ). ${ }^{9} \mathrm{He}$ was therefore discharged on oral posaconazole alone, on the advice of the infectious disease physicians. He was also discharged on insulin, and diabetic follow up was arranged. Out-patient MRI scans were arranged for four weeks and one year post-discharge.

Upon review in the clinic, the patient was well; however, he did mention cachosmia and discomfort from his eye patch, which was subsequently reviewed by the prosthetics department. He was recommended to continue nasal douching to keep the cavity clean, and was advised that he may need further decrusting if the cachosmia did not improve. The initial (four week) out-patient MRI revealed that the disease was static. The MRI taken one year postdischarge did not show recurrence. The patient was therefore advised to continue posaconazole treatment, and was referred back to his local hospital for follow up, with provisional arrangements for liaison with the infectious disease team if needed. 


\section{Discussion}

Based on clinical and histopathological findings, fungal sinusitis has been classified into three categories: invasive, non-invasive and allergic. ${ }^{2}$ Invasive sinusitis can be further subdivided into granulomatous, acute fulminant and chronic invasive types. ${ }^{1}$ The diagnostic criteria proposed for invasive fungal sinusitis include radiological confirmation of sinusitis, and histopathological evidence of hyphal forms within sinus mucosa, submucosa, blood vessels or bone.

Mucormycosis can manifest as six different syndromes: rhinocerebral, pulmonary, gastrointestinal, central nervous system, subcutaneous and disseminated. ${ }^{3}$ Rhinocerebral mucormycosis falls under the category of acute fulminant invasive sinusitis. ${ }^{1,2}$ This particular form is characterised by a painless, necrotic septal ulcer, sinusitis and rapid intracranial spread with a high mortality rate. The syndrome develops most commonly in individuals with poorly controlled diabetes, as in our patient. ${ }^{1-4,10-12}$ Immunocompromise is the main predisposing factor, and this may be congenital, acquired (e.g. acquired immunodeficiency syndrome) or drug-induced (e.g. steroids, or following chemotherapy or immune suppression after organ transplantation). ${ }^{12}$ Other known predisposing factors include haematological malignancy, severe burns, renal disease, iron overload and the use of desferoxamine. ${ }^{1-3,10,11}$ Acidosis and hyperglycaemia are ideal environments for fungal growth.

The infection can spread from the nasal mucosa into the paranasal sinuses (specifically the ethmoid and maxillary sinuses) and the brain. The exact pathogenesis of spread is not clear. However, it is believed that the pterygopalatine fossa plays a key role in providing routes for extension of the disease. ${ }^{11}$ There are several foramina that connect this space to others: the inferior orbital fissure to the retrobulbar space; the pterygo-maxillary fissure to the inferior temporal fossa and masticator space; the sphenopalatine foramen to the nasal cavity; the foramen rotundum and pterygoid canal to the middle cranial fossa; and finally the greater palatine canal to the palate. ${ }^{6}$ Involvement of the various nerves and vessels in the region gives rise to various clinical manifestations, such as the facial hypoaesthesia, diplopia and proptosis observed in our patient.

Medical treatment of infective rhinocerebral mucormycosis has been well documented, in the form of the polyene antifungal drug amphotericin $\mathrm{B}^{2-4,10,12-14}$ Toxic renal and systemic side effects are common, due to the high doses and prolonged treatment duration required. Safer and more effective drugs are therefore needed. Several lipid formulations of amphotericin B have been developed, including the liposomal amphotericin B (AmBisome) used to treat our patient. ${ }^{3}$ Lipid formulations of amphotericin B appear not only to be safer but also more efficacious in mucormycosis therapy. ${ }^{4,15}$ In the case of our patient, however, drug-induced transaminitis occurred. The liposomal amphotericin B may also have contributed to the patient's sensorineural hearing loss; however, the unilateral nature of the hearing loss would seem to make this unlikely. This hearing loss may also have been due to disease progression; however, there was no convincing radiological evidence of this. The end result was that the liposomal amphotericin B dosage was reduced to a minimum and a second antifungal drug, posaconazole, was added.

Posaconazole is a newer, orally bioavailable, extendedspectrum, triazole antifungal active against a range of yeasts and hyaline moulds. ${ }^{12,16}$ However, in contrast to previous azole antifungals such as fluconazole and voriconazole, it also demonstrates enhanced in vitro activity against mucorales. ${ }^{10}$ However, there are concerns about achieving reliable therapeutic levels in vivo to treat mucormycosis. ${ }^{17}$ Further data from models of neutropenic murine mucormycosis have failed to prove the efficacy of posaconazole as monotherapy. ${ }^{18}$ It is therefore not currently recommended as primary treatment for mucormycosis.

In contrast, in patients who fail to respond to polyenes, or who are intolerant of them (as in our case), posaconazole may be a reasonable option. A study of 24 patients with zygomycosis, 19 refractory to their therapy and five intolerant, showed that 19 (79 per cent) of the 24 had a complete or partial response to posaconazole. ${ }^{6}$ Four out of the five intolerant patients had a complete or partial response.

A later, retrospective study involved 91 patients with proven $(n=69)$ or probable $(n=22)$ mucormycosis, who were refractory to $(n=48)$ or intolerant of $(n=10)$ prior antifungal therapy, or both $(n=33)^{7}$. The majority had previously received lipid formulations of amphotericin $\mathrm{B}$, and all were given posaconazole for at least 30 days. Fifty-five patients (60 per cent) experienced a complete or partial response at 12 weeks' follow up. Nineteen patients (21 per cent) had stable disease, and 15 (17 per cent) experienced treatment failure.

Posaconazole is generally well tolerated, with few significant adverse effects. ${ }^{17}$ This allowed us to discharge our patient on long-term posaconazole, to be followed up locally. Effective treatment of mucormycosis with liposomal amphotericin B generally requires doses in excess of $5 \mathrm{mg} /$ $\mathrm{kg}$ (one study found a median of $7 \mathrm{mg} / \mathrm{kg}$ ). ${ }^{5}$ Toxic effects typically occur with doses in excess of $3 \mathrm{mg} / \mathrm{kg}$, and are more common with doses of $5-10 \mathrm{mg} / \mathrm{kg} .{ }^{9}$ Therefore, combination therapy with liposomal amphotericin B and posaconazole may permit the use of a lower dose of liposomal amphotericin $\mathrm{B}$, hence reducing the risk of side effects while still being effective. Whilst there is insufficient data to recommend the use of posaconazole as first-line treatment, the studies and theories described above highlight the need for further research on the use of this drug in the treatment of mucormycosis.

\section{- Infective rhinocerebral mucormycosis is an aggressive disease more prevalent in} immunocompromised hosts

- The mainstay of treatment is surgical debridement with concomittant systemic antifungal therapy

- The polyene antifungal drug amphotericin $B$ is an effective treatment option, but may have toxic systemic side effects

- Posaconazole is an antifungal that can be considered for salvage therapy of mucormycosis

Surgical treatment of rhinocerebral mucormycosis, in the form of debridement, is also fundamental, as medical management alone is associated with a poor outcome. ${ }^{3}$ The amount of debridement is guided by the patient's overall condition, the extent and aggressiveness of invasion, and the response to initial treatment. Orbital exenteration is not mandatory in all cases with evidence of orbital disease, but it was necessary in our patient. ${ }^{3,4}$ Due to the pathogenesis of mucormycosis spread, it has been proposed that the pterygopalatine fossa is a key anatomical region to debride. $^{11}$

Hyperbaric oxygen therapy has been shown to be a useful adjunct in the treatment of invasive fungal infections. ${ }^{19}$ Unfortunately, our patient suffered panic attacks upon attempting this form of treatment, so it was abandoned. 
Rhinocerebral mucormycosis remains an aggressive disease with a high mortality rate. With the increasing prevalence of diabetes and immunosuppressive conditions, especially associated with the rising number of organ transplants, opportunistic fungal infections will become more prevalent. Therapy with triazoles is a key advancement in the treatment of invasive fungal infection. However, the need persists for early recognition, correction of underlying disorders and urgent, effective treatment.

\section{References}

1 de Shazo RD, O'Brien M, Chaplin K, Soto-Aguilar M, Gardner L, Swain R. A new classification and diagnositic criteria for invasive fungal sinusitis. Arch Otolaryngol Head Neck Surg 1997;123:1181-8

2 Morgan J, Wamock DW. Fungi. In: Jones NJ, ed. ScottBrown's Otolaryngology, Head and Neck Surgery. Oxford: Arnold, 2008;1:213-17

3 Peterson KL, Wang M, Canalis RF, Abemayor E. Rhinocerebral mucormycosis: evolution of the disease and treatment options. Laryngoscope 1997;107:855-62

4 Strasser MD, Kennedy RJ, Adam RD. Rhinocerebral mucormycosis: therapy with amphotericin B lipid complex. Arch Intern Med 1996;156:337-9

5 Kontoyiannis DP, Wessel VC, Bodey GP, Rolston KV. Zygomycosis in the 1990s in a tertiary-care cancer center. Clin Infect Dis 2000;30:851-6

6 Greenberg RN, Mullane K, van Burik JA, Raad I, Abzug MJ, Anstead G et al. Posaconazole as salvage therapy for zygomycosis. Antimicrob Agents Chemother 2006;50: $126-33$

7 Van Burik JA, Hare R, Solomon H, Corrado ML, Kontoyiannis DP. Posaconazole is effective as salvage therapy in zygomycosis: a retrospective summary of 91 cases. Clin Infect Dis 2006;43:376

8 Rodriguez MM, Serena C, Mariné M, Pastor FJ, Guarro J. Posaconazole combined with amphotericin $\mathrm{B}$, an effective therapy for a murine disseminated infection caused by Rhizopus oryzae. Antimicrob Agents Chemother 2008;52: 3786-8

9 AmBisome. Summary of Product Characteristics. In: http:// emc.medicines.org.uk/medicine/1236/SPC/AmBisome/ [30 July 2009]

10 Greenberg RN, Scott LI, Vaughn HH, Ribes JA Zygomycosis (mucormycosis): emerging clinical importance and new treatments. Curr Opin Infect Dis 2004;17 $517-25$
11 Hosseini SMS, Borghei P. Rhinocerebral mucormycosis: pathways of spread. Eur Arch Otorhinolaryngol 2005;262: 932-8

12 Tobon AM, Arango M, Fernandex D, Restrepo A. Mucormycosis (zygomycosis) in a heart-kidney transplant recipient: recovery after posaconazole therapy. Clin Infect Dis 2003;36:1488-91

13 Pardal Refoyo JL, Chocarro Martinez A, Brezmes Valdivieso MF, Parra Pérez C. Treatment review in rhinocerebral mucormycosis [in Spanish]. An Otorrinolaringol Ibero Am 1998;25:45-56

14 Streppel M, Bachmann G, Arnold G, Damm M, Stennert E. Successful treatment of an invasive aspergillosis of the skull base and paranasal sinuses with liposomal amphotericin B and itraconazole. Ann Otol Rhinol Laryngol 1999;108: 205-7

15 Walsh TJ, Hiemenz JW, Seibel NL, Perfect JR, Horwith G, Lee L et al. Amphotericin B lipid complex for invasive fungal infections: analysis of safety and efficacy in 556 cases. Clin Infect Dis 1998;26:1383-96

16 Sabatelli F, Patel R, Mann PA, Mendrick CA, Norris CC, Hare $\mathrm{R}$ et al. In vitro activities of posaconazole, fluconazole, itraconazole, voriconazole, and amphotericin B against a large collection of clinically important molds and yeasts. Antimicrob Agents Chemother 2006;50:2009-15

17 Ullmann AJ, Cornely OA, Burchardt A, Hachem R, Kontoyiannis DP, Töpelt K et al. Pharmacokinetics, safety, and efficacy of posaconazole in patients with persistent febrile neutropenia or refractory invasive fungal infection. Antimicrob Agents Chemother 2006;50:658-66

18 Ibrahim AS, Gebremariam T, Schwartz JA, Edwards JE Jr, Spellberg B. Posaconazole mono- or combination therapy for treatment of murine zygomycosis. Antimicrob Agents Chemother 2009;53:772-5

19 Garcia-Covarrubias L, Barrat DM, Bartlett R, Metzinger S, Van Meter K. Invasive aspergillosis treated with adjunctive hyperbaric oxygenation: a retrospective clinical series at a single institution. South Med J 2002;95:450-6

Address for correspondence:

Mr K Kulendra,

25 Buxton Drive,

New Malden KT3 3UX, UK.

Fax: 02083950117

E-mail:knk79@blueyonder.co.uk

Mr K Kulendra takes responsibility for the integrity

of the content of the paper.

Competing interests: None declared 